\section{Antiplatelet therapy is not a safer alternative to oral anticoagulants, even in older hospital-discharged patients with atrial fibrillation}

\author{
Mario Bo, ${ }^{1}$ Yolanda Falcone, ${ }^{1}$ \\ Enrica Grisoglio, ${ }^{1}$ Margherita Marchetti, \\ Federica Li Puma, ${ }^{1}$ Marina lacovino, ${ }^{1}$ \\ Enrico Brunetti, ${ }^{1}$ Gianfranco Fonte ${ }^{2}$ \\ 'Department of Geriatrics, Città della \\ Salute e della Scienza - Molinette \\ Hospital, University of Torino; ${ }^{2}$ Unit of \\ Post-Acute Care, Città della Salute e della \\ Scienza, University of Torino, Italy
}

\section{Abstract}

Although oral anticoagulant therapy (OAT) is recommended for patients with atrial fibrillation (AF), it is widely underused among older patients, who are frequently prescribed antiplatelet therapy (APT) instead. We assessed mortality and incidence of ischemic and hemorrhagic events according to prescription of OAT or APT in older medical in-patients with $\mathrm{AF}$ discharged from hospital.

Stroke and bleeding risk were evaluated using the $\mathrm{CHA}_{2} \mathrm{DS}_{2}$-VASC (Congestive heart failurelleft ventricular dysfunction, Hypertension, Aged $\geq 75$ years, Diabetes Mellitus, Stroke/transient ischemic attack/systemic embolism, Vascular Disease, Aged 65-74 years, Sex Category) and HAS-BLED (Hypertension, Abnormal renalliver function, Stroke, Bleeding history or predisposition, Labile international normalized ratio, Elderly, Drugs/alcohol concomitantly) scores. Comorbidity, cognitive status and functional autonomy were assessed using standardized scales. Association of OAT and APT with overall mortality, ischemic stroke and bleeding events was evaluated through multivariate analysis and propensity score matching.

During a mean follow-up period of 11 months 384 of the 962 patients discharged (mean age $82.9 \pm 6.6$ years, $59.1 \%$ female) died (39.9\%), 66 had an ischemic stroke and 49 experienced a major bleeding event. Compared with APT, OAT was associated with reduced overall mortality after multivariate analysis [odds ratio (OR) 0.62 , confidence interval (CI): 0.46-0.83] and after propensity score matched analysis (OR 0.65, CI: 0.52-0.82, $\mathrm{P}=0.0004$ ), with a not significant reduced incidence of total and fatal ischemic stroke, and without increase in total, intracranial, major and fatal bleedings.

In a sample of older AF patients with poor health status, OAT was associated with reduced mortality, without evidence of a significant increase in major or fatal bleedings.

\section{Introduction}

Incidence and prevalence of atrial fibrillation (AF) increase with advancing age. ${ }^{1}$ Although oral anticoagulant therapy (OAT) has been showed to be effective for stroke prevention in older patients with $\mathrm{AF}^{2-4}$ this therapy is widely underused particularly in the oldest-old, who should derive the greatest benefit from anticoagulant therapy. ${ }^{5-9} \mathrm{AF}$ in elderly patients is frequently diagnosed during hospital stay, and it has been demonstrated that many hospitalized older patients, who might not be optimal candidates for OAT in reason of advanced age, perceived concerns around significant bleeding risks, poor health conditions, increased comorbidities, poor functional autonomy and reduced life-expectancy, are prescribed anti-platelets therapy (APT) instead. ${ }^{5-13}$ Indeed, it is a long-standing common belief among many physicians that APT, although offering less protection against cardio-embolic stroke, may represent an overall safer alternative to OAT for older patients with poor health status. As a result, recent studies demonstrate that there is a persistent attitude to prescribe APT instead of OAT in older patients with AF in several clinical settings, and that this practice is increasingly common among the oldest old. ${ }^{5-7,9,10,14-16}$

Recently, we reported that among older patients with AF discharged from an acute geriatric ward with high one-year mortality, OAT was associated with a significant reduction of overall mortality, ${ }^{17}$ and very similar results we observed in a prospective study among older patients discharged from acute medical and geriatric wards, with OAT being associated with lower overall mortality and ischemic stroke incidence. ${ }^{9}$ However, none of these studies specifically addressed a direct comparison between OAT and APT in these older patients. Therefore, to evaluate whether APT may represent a reasonable antithrombotic therapeutic option in AF at least among these older patients with poor health status, we assessed overall mortality and incidence of ischemic and hemorrhagic events, in older medical patients with AF according to the prescription at discharge of OAT or APT.

\section{Materials and Methods}

The patients' sample for the present study was derived from the two studies, which have been cited above. The retrospective study ${ }^{17}$
Correspondence: Yolanda Falcone, Department of Geriatrics, Città della Salute e della Scienza Molinette Hospital, University of Torino, Corso Bramante 88, 10126, Torino, Italy.

Tel.: +39.011.6336660 - Fax: +39.011 .6335263 .

E-mail: yolanda.falcone@yahoo.it

Key words: Atrial fibrillation; elderly; oral anticoagulant therapy; antiplatelet therapy.

Conflict of interest: the authors declare no potential conflict of interest.

Contributions: MB, GF, data first collection and analysis; MB, GF, YF, study design; MI, EG, FLP, $\mathrm{EB}$, study conduction and data acquisition; GF, $\mathrm{MB}, \mathrm{MM}$, data management and analysis; MB, MM, data interpretation; MB, YF, MM, FLP, manuscript preparation. All authors approved the manuscript final version to be published.

Received for publication: 1 July 2016. Revision received: 3 September 2016.

Accepted for publication: 6 September 2016.

This work is licensed under a Creative Commons Attribution-NonCommercial 4.0 International License (CC BY-NC 4.0).

(C) Copyright M. Bo et al., 2016

Licensee PAGEPress, Italy

Geriatric Care 2016; 2:6120

doi:10.4081/gc.2016.6120

included patients $\geq 65$ years of age discharged in the period 2010-2013 from the Acute Geriatric Ward at the Città della Salute e della Scienza - Molinette Hospital (a university teaching hospital in Torino, northern Italy) with a primary or secondary diagnosis of AF [code 427.31 of the International Classification of Diseases, Ninth Revision (ICD-9)], who were identified from the electronic discharge database. The cohort of prospective study ${ }^{9}$ was identified in the period from October 2013 to August 2014 among patients aged $\geq 65$ years with AF (code 427.31 ICD-9) discharged from the following clinical units: Geriatria e Malattie Metaboliche dell'Osso, Medicina Interna 1U, Medicina Interna 3U, Medicina Interna 4U, Medicina Interna 6, Medicina Interna DEA (A.O.U. Città della Salute e della Scienza, Torino Molinette), Geriatria (A.O.U.S. Luigi Gonzaga, Orbassano), and Geriatria (A.S.O. S. Croce e Carle, Cuneo).

AF was defined paroxysmal, persistent or permanent according to current international recommendations. Individual stroke and bleeding risk were evaluated according to the $\mathrm{CHA}_{2} \mathrm{DS}_{2}$-VASC (Congestive heart failure/left ventricular dysfunction, Hypertension, Aged $\geq 75$ years, Diabetes Mellitus, Stroke/transient ischemic attack/systemic embolism, Vascular Disease, Aged 65-74 years, Sex Category $)^{18}$ and 
HAS-BLED (Hypertension, Abnormal renaV liver function, Stroke, Bleeding history or predisposition, Labile international normalized ratio, Elderly, Drugs/alcohol concomitantly) ${ }^{19}$ scores, respectively.

All patients enrolled underwent a full comprehensive geriatric assessment, including indexes of comorbidity and global physical health (Charlson index) ${ }^{20}$ cognitive status [short portable mental status questionnaire (SPMSQ) $],{ }^{21}$ and functional autonomy (activities of daily living (ADL); instrumental activities of daily living scale (IADL)], ${ }^{22,23}$ which were also included for analysis. Patients were defined not to have cognitive impairment with SPMSQ score $0-2$; SPMSQ score of 3-4, 5-7 and $\geq 8$, identified mild, moderate and severe cognitive impairment, respectively. Patients were defined partially or totally dependent in basic daily activities with ADL score of 1-2 and $\geq 3$, respectively. Patients were defined dependent in instrumental daily activities with a IADL score of $9 / 14$ or less. For each patient, creatinine at discharge was also recorded, and estimated glomerular filtration rate was calculated according to the Chronic Kidney Disease Epidemiology Collaboration (CKD-EPI) formula. ${ }^{24}$

In both previous studies, antithrombotic therapy at discharge was recorded, according to the following classes: OAT only, single APT, double APT, combined double or triple anticoagulantantiplatelet therapy, and other, mainly represented by low molecular weight heparin. In the present study, to address the conundrum of clinical practice (anticoagulant or aspirin?) only patients discharged with OAT or with single APT were included. Indeed, single APT (mainly aspirin and less frequently ticlopidine or clopidogrel) is the usual alternative to OAT in common clinical practice for older AF patients.

In both studies follow-up was conducted by four geriatric post-graduate students under the supervision of a senior geriatrician through telephone interview with patients or usual caregivers in patients living at home, and through review of medical charts in patients resident in long-term facilities. Death, ischemic and hemorrhagic events, and switch of antithrombotic treatment were investigated. Among patients reporting any hospitalization or suspected clinical event of interest a thorough review of clinical documentation, medical charts, in-patient hospital discharges and death certificate was performed by a senior geriatrician. Death and its causes were assessed from death certificates, patients' hospital records and information from patient's general practitioner.

Ischemic and hemorrhagic strokes, according to American Heart Association/American Stroke Association (AHA/ASA) definition ${ }^{25}$ were recorded. We categorized bleedings as major or minor events. Major bleeding was defined as fatal bleeding, and/or symptomatic bleeding in a critical area or organ, and/or bleeding causing a fall in hemoglobin level of $20 \mathrm{~g} / \mathrm{L}(1.24 \mathrm{mmol} / \mathrm{L})$ or more, or leading to transfusion of two or more units of whole blood or red cells, and/or bleeding causing patient's hospitalization according to current international recommendations. ${ }^{26}$

The study was conducted according to the principles of the Declaration of Helsinky Title 45, U.S. Code of Federal Regulations, Part 46, Protection of Human Subjects, Revised November 13, 2001, effective December 13, 2001, and according to the Recommendations Guiding Physicians in Biomedical research Involving Human Subjects.

\section{Statistical analysis}

In the present study we compared main clinical outcomes, including overall mortality, ischemic and hemorrhagic stroke, fatal and non-fatal major bleeding, and minor bleedings, in patients who were discharged with a prescription for OAT and in those who were discharged on APT. Absolute and relative frequencies of dichotomous and categorical variables and mean and relative distribution of continuous variables were calculated. Associations between variables and fatal and non-fatal clini- cal end-points were evaluated using analysis of variance, Chi-square and Mann-Whitney test, and then using a logistic regression model (forward stepwise method) to evaluate significant independent associations. In addition, a propensity score matching using a 1:1 nearestneighbor-matching algorithm with a \pm 0.02 caliper and no replacement (yielding 236 propensity score matched observations), was used to evaluate clinical outcomes after adjustment for significant differences in patient baseline characteristics. Matched sample comparisons were performed with McNemar Test, Paired T test and Wilcoxon test. ${ }^{27}$

\section{Results}

The main clinical characteristics of the 962 patients studied are presented in Table 1 . Mean age of patients was $82.9 \pm 6.6$ years (with $88.7 \%$ of patients being 75 years of age or older) and $59.1 \%$ were females. Mean $\mathrm{CHA}_{2} \mathrm{DS}_{2}$-VASC and HAS-BLED scores were $4.7 \pm 1.4$ and $2.3 \pm 1.0$, respectively. Cognitive impairment was observed in $49.7 \%$ of patients, and functional dependence in ADL and IADL in

Table 1. Demographic and clinical variables, and antithrombotic therapy at discharge, in the overall sample $(n=962)$.

\begin{tabular}{|c|c|}
\hline Age, years, mean \pm standard deviation & $82.9 \pm 6.6$ \\
\hline Age $\geq 75$ years, $n(\%)$ & $853(88.7 \%)$ \\
\hline Female, $\mathrm{n}(\%)$ & $569(59.1 \%)$ \\
\hline Persistent/permanent AF, n (\%) & $725(75.4 \%)$ \\
\hline $\mathrm{CHA}_{2} \mathrm{DS}_{2}-\mathrm{VASC}$, mean \pm standard deviation & $4.7 \pm 1.4$ \\
\hline HAS-BLED, mean \pm standard deviation & $2.3 \pm 1.0$ \\
\hline HAS-BLED $\geq 3, \mathrm{n}(\%)$ & $356(37.0 \%)$ \\
\hline Charlson comorbidity index, mean \pm standard deviation & $5.9 \pm 2.8$ \\
\hline Charlson $>5, \mathrm{n}(\%)$ & $567(58.9 \%)$ \\
\hline Serum creatinine, $\mathrm{mg} / \mathrm{dL}$, median $\left(25^{\circ}-75^{\circ}\right)$ & $1.07(0.87-1.44)$ \\
\hline eGFR (CKD-EPI) & $53.9 \pm 21.1$ \\
\hline eGFR (CKD-EPI) <60 mL/min, n (\%) & $587(61.0 \%)$ \\
\hline ADL dependent, $n(\%)$ & $466(48.4 \%)$ \\
\hline IADL dependent, $n(\%)$ & $639(66.4 \%)$ \\
\hline Cognitive impairment, n (\%) & $478(49.7 \%)$ \\
\hline Number of drugs at discharge, median $\left(25^{\circ}-75^{\circ}\right)$ & $8(6-10)$ \\
\hline $\begin{array}{l}\text { Antithrombotic therapy at discharge: } \\
\text { OAT, } n \text { (\%) } \\
\text { Warfarin/NKAs } \\
\text { Dabigatran } \\
\text { Apixaban } \\
\text { Rivaroxaban } \\
\text { APT, n (\%) } \\
\end{array}$ & $\begin{array}{c}520(54.1 \%) \\
487(93.6 \%) \\
19(3.6 \%) \\
9(1.7 \%) \\
5(0.9 \%) \\
442(45.9 \%)\end{array}$ \\
\hline \multicolumn{2}{|c|}{$\begin{array}{l}\text { AF, atrial fibrillation; } \text { CHA }_{2} \mathrm{DS}_{2} \text {-VASC, Congestive heart failure/left ventricular dysfunction, Hypertension, Aged>=75 years, Diabetes Mellitus, } \\
\text { Stroke/transient ischemic attack/systemic embolism, Vascular Disease, Aged 65-74 years, Sex Category; HAS-BLED, Hypertension, Abnormal } \\
\text { renal/iver function, Stroke, Bleeding history or predisposition, Labile international normalized ratio, Elderly, Drugs/alcohol concomitantly } \\
\text { eGFR, estimated glomerular filtration rate; CKD-EPI, Chronic Kidney Disease Epidemiology Collaboration; ADL, activities of daily living; IADL, } \\
\text { instrumental activities of daily living scale; OAT, oral anticoagulant therapy; VKAs, vitamin K antagonists; APT, anti platelet therapy. }\end{array}$} \\
\hline
\end{tabular}


$48.4 \%$ and $66.4 \%$ of patients, respectively; the mean comorbidity index was $5.9 \pm 2.8$. Overall, 520 patients were discharged with OAT [ $93.6 \%$ receiving vitamin $\mathrm{K}$ antagonists (VKAs)], and 442 received APT.

During a mean follow-up period of 11 months 384 patients died, 66 had an ischemic stroke and 49 major bleedings occurred. Mortality, and ischemic and hemorrhagic events, in the overall sample and according to prescription of OAT and APT, are reported in Table 2. Patients discharged with OAT had lower incidence of overall mortality $(29.8 \% \mathrm{vs}$ $51.8 \%, \mathrm{P}=0.0000)$ and ischemic stroke (5.0\% vs $9.0 \%, \mathrm{P}=0.013$ ) than patients treated with APT. After multivariate analysis, OAT at discharge was associated with reduced overall mortality [odds ratio (OR) 0.62 , confidence interval (CI): $0.46-0.83, \mathrm{P}=0.0013$ ], whereas increasing age, loss of functional autonomy and increasing comorbidity score were associated with increased odd of death (Table 3 ).

Analysis of clinical events in 236 propensity score matched observations showed that OAT was associated with lower overall death rate (OR 0.65, CI: 0.52-0.82, $\mathrm{P}=0.0004$ ), as well as with a not significant trend to a reduced inci- dence of total and fatal ischemic stroke, whereas fatal, intracranial, major and fatal bleedings overall were not significantly more frequent among OAT treated patients than in those receiving APT (Table 4).

\section{Discussion}

Despite most international guidelines strongly recommend use of OAT and discourage use of aspirin for patients with $\mathrm{AF}$ and high cardio-embolic risk (that is a $\mathrm{CHA}_{2} \mathrm{DS}_{2}$ VASC score greater than 1), several contemporary studies clearly demonstrate a persistent reluctance to prescribe OAT, particularly among older and vulnerable patients, who are often treated with aspirin or other anti-platelet drugs. ${ }^{6,9,10}$ To evaluate whether APT may represent a reasonable antithrombotic therapeutic option in $\mathrm{AF}$ at least among these older patients with poor health status, we assessed overall mortality and incidence of ischemic and hemorrhagic events, in older medical patients with AF discharged from hospital with a prescription for OAT or APT.
In this sample of patients with poor health status and high post-discharge mortality, we observed a very high incidence of ischemic stroke, with increased fatality rates. Within the intrinsic limitations of an observational cohort study, our findings show that compared with APT, OAT was associated with reduced overall mortality, without evidence of a significant increased risk of major bleedings. The reduced overall mortality associated with OAT remained significant after multivariate analysis and propensity score matching, whereas the reduction in incidence and mortality for ischemic stroke were not significant after propensity score matching. Importantly, OAT use was not associated with a significant increase in major bleeding events. Our findings of efficacy and safety of OAT appear to be in keeping with results of BAFTA study ${ }^{2}$ and with a very recent paper reporting efficacy and safety of apixaban compared with aspirin among older outpatients enrolled in the AVERROES trial. ${ }^{28}$ However, compared with these studies in community-dwelling older subjects, we observed a greater incidence of ischemic stroke and bleedings in our cohort of patients, as it is reasonable to expect among very elderly

Table 2. Mortality and major clinical events at follow-up according to antithrombotic therapy at discharge.

\begin{tabular}{|c|c|c|c|c|c|}
\hline Clinical events & $\begin{array}{c}\text { Total sample } \\
\quad(n=962)\end{array}$ & $\begin{array}{c}\text { OAT } \\
(\mathrm{n}=520)\end{array}$ & $\begin{array}{c}\text { APT } \\
(n=442)\end{array}$ & Chi square & $\mathbf{P}$ \\
\hline Overall mortality, n (\%) & $384(39.9 \%)$ & $155(29.8 \%)$ & $229(51.8 \%)$ & 48.225 & 0.00000 \\
\hline Total ischemic stroke, $\mathrm{n}(\%)$ & $66(6.9 \%)$ & $26(5.0 \%)$ & $40(9.0 \%)$ & 6.132 & 0.01327 \\
\hline Fatal ischemic stroke, $\mathrm{n}(\%)$ & $28(2.9 \%)$ & $10(1.9 \%)$ & $18(4.1 \%)$ & 4.115 & 0.0425 \\
\hline Ischemic events, other sites, n (\%) & $50(5.2 \%)$ & $25(4.8 \%)$ & $25(5.7 \%)$ & 0.349 & 0.55467 \\
\hline Hemorrhagic stroke/intracranial bleeding, n (\%) & $9(0.9 \%)$ & $6(1.15 \%)$ & $3(0.68 \%)$ & 0.581 & 0.44556 \\
\hline Major extra-cranial bleeding, $\mathrm{n}(\%)$ & $40(4.1 \%)$ & $25(4.8 \%)$ & $15(3.4 \%)$ & 1.198 & 0.27357 \\
\hline Major bleeding, total, n (\%) & $49(5.1 \%)$ & $31(6.0 \%)$ & $18(4.1 \%)$ & 1.763 & 0.18414 \\
\hline Fatal bleeding, n (\%) & $11(1.1 \%)$ & $6(1.1 \%)$ & $5(1.1 \%)$ & 0.001 & 0.9738 \\
\hline Minor bleeding, n (\%) & $56(5.8 \%)$ & $37(7.1 \%)$ & $19(4.3 \%)$ & 3.457 & 0.06296 \\
\hline
\end{tabular}

OAT, oral anticoagulant therapy; APT, anti platelet therapy.

Table 3. Variables associated with mortality and ischemic stroke (multivariate analysis). No significant associations were found for other major clinical events investigated.

\begin{tabular}{|c|c|c|c|c|}
\hline & B & SE & $\mathbf{P}$ & OR (CI 95\%) \\
\hline \multicolumn{5}{|l|}{ Overall mortality } \\
\hline Age & 0.07 & 0.01 & 0.0000 & 1.07 (1.04-1.09) \\
\hline Charlson comorbidity index & 0.15 & 0.03 & 0.0000 & $1.16(1.10-1.22)$ \\
\hline $\mathrm{ADL} \geq 2$-dependent & 0.78 & 0.15 & 0.0000 & $2.19(1.64-2.92)$ \\
\hline OAT at discharge & -0.48 & 0.15 & 0.0013 & $0.62(0.46-0.83)$ \\
\hline \multicolumn{5}{|l|}{ Ischemic stroke } \\
\hline Charlson comorbidity index & 0.14 & 0.05 & 0.0041 & $1.15(1.04-1.26)$ \\
\hline $\mathrm{CHAD}_{2} \mathrm{DS}_{2}$-VASC score & 0.22 & 0.10 & 0.0199 & $1.25(1.04-1.51)$ \\
\hline
\end{tabular}

SE, standard error; OR, odds ratio; Cl, confidence intereval; $\mathrm{ADL}$, activities of daily living; OAT, oral anticoagulant therapy; $\mathrm{CHA}_{2} \mathrm{DS}$-VASC, Congestive heart failure/left ventricular dysfunction, Hypertension, Aged $>=75$ years, Diabetes Mellitus, Stroke/transient ischemic attack/systemic embolism, Vascular Disease, Aged 65-74 years, Sex Category. 
hospital-discharged high-risk medical patients. Indeed, the incidence of major bleeding among OAT treated patients was also slightly higher than the bleeding rates reported in a recent national survey on anti-coagulated elderly AF outpatients. ${ }^{29}$ However, the magnitude of reduction in incidence of ischemic stroke we observed among patients treated with OAT compared to APT treated patients is in keeping with previous studies, ${ }^{2-4}$ and the incidence of fatal ischemic strokes was also roughly halved among patients treated with OAT.

The population we studied is important for several reasons, including the high AF prevalence, the high post-discharge mortality ${ }^{30}$ the under-representation of such patients in prior trials of OAT for AF, the common reluctance in daily clinical practice to prescribe 0AT, and the scant evidence of benefit of anticoagulation in such elderly frail patients. In our view, present findings have clinical implications, potentially contributing to reduce physicians' reluctance to prescribe anticoagulants in these patients.

Some limitations of this study must be addressed. The main weakness is clearly the potential for selection bias, which is inherent to the observational nature of the cohorts studied. Compared with patients treated with APT, those discharged with OAT are younger and have better functional and health status, conditions that are known to correlate with better survival. In order to mitigate this potential bias we firstly evaluated whether OAT or APT were independently associated with fatal and nonfatal clinical end-points (mortality and ischemic/hemorrhagic events) and, secondly, we used a propensity score matching in order to disentangle the net effect of OAT $v s$ APT from other confounding variables. However, despite these precautions and the comprehensive geriatric assessment, multivariable analysis and propensity score matching might not account for other unknown or unmeasured influent variables. As in other observational studies a second limitation is that, despite most of patients were still on prescribed anticoagulant treatment at the follow up interview or at the censored event, we could not evaluate therapeutic adherence and time in therapeutic range in patients receiving warfarin. However, in our view this limitation does not diminish the external validity of present findings, which aim to represent the effect of OAT among older real-world patients rather than in the more comfortable setting of randomized trial with strictly monitored patients. Finally, as stated, direct oral anticoagulants were not available in our country until 2013; therefore only few patients received a direct oral anticoagulant (33 patients), most of patients being treated with VKAs (487 patients). However, results did not change when analysis was limited to patients treated with VKAs. Current and future observational studies involving older patients treated with new direct oral anticoagulants (which are associated with a reduced intracranial bleeding risk) may therefore further move the benefit balance in favor of OAT among these frail older medical patients with AF.

Some strengths of this study should also be considered. The enrollment of patients in several medical and geriatric wards should reassure about the possibility of generalization of our findings. Secondly, due to the high proportion of older patients (roughly $90 \%$ of patients in the present study were aged 75 year or older) in the sample studied, our findings might shed some light on the often-neglected world of AF older patients in medical and geriatric units. Thirdly, patients' evaluation

Table 4. Demographic and clinical variables, mortality and major clinical events, before and after propensity score matching by treatment groups (oral anticoagulant and anti platelet therapy).

\begin{tabular}{|c|c|c|c|c|c|c|}
\hline \multirow[b]{2}{*}{ Baseline clinical variables } & \multicolumn{3}{|c|}{ Before propensity score matching } & \multicolumn{3}{|c|}{ After propensity score matching } \\
\hline & $\begin{array}{c}\text { OAT } \\
(n=520)\end{array}$ & $\begin{array}{c}\text { APT } \\
(\mathrm{n}=442)\end{array}$ & $\mathbf{P}$ & $\begin{array}{c}\text { OAT } \\
(\mathrm{n}=236)\end{array}$ & $\begin{array}{c}\text { APT } \\
(n=236)\end{array}$ & $\mathbf{P}$ \\
\hline Age, mean \pm standard deviation & $81.3 \pm 6.04$ & $84.7 \pm 6.74$ & 0.0000 & $83.4 \pm 6.0$ & $83.8 \pm 6.6$ & 0.414 \\
\hline Female, $\mathrm{n}(\%)$ & $307(59.0 \%)$ & $262(59.3 \%)$ & 0.9404 & $133(56.4 \%)$ & $135(57.2 \%)$ & 0.9279 \\
\hline Persistent/permanent AF, n (\%) & $430(82.7 \%)$ & $295(66.7 \%)$ & 0.0000 & $171(72.5 \%)$ & $169(71.6 \%)$ & 0.9195 \\
\hline Serum creatinine, $\mathrm{mg} / \mathrm{dL}$, mean \pm standard deviation & $1.25 \pm 0.62$ & $1.24 \pm 0.60$ & 0.9148 & $1.26 \pm 0.57$ & $1.24 \pm 0.61$ & 0.745 \\
\hline $\mathrm{CHA}_{2} \mathrm{DS}_{2}$-VASC score, mean \pm standard deviation & $4.71 \pm 1.30$ & $4.81 \pm 1.42$ & 0.2564 & $4.75 \pm 1.34$ & $4.74 \pm 1.46$ & 0.897 \\
\hline HAS-BLED score, mean \pm standard deviation & $2.08 \pm 0.98$ & $2.49 \pm 1.06$ & 0.0000 & $2.41 \pm 1.09$ & $2.31 \pm 0.97$ & 0.308 \\
\hline Charlson comorbidity index, mean \pm standard deviation & $5.35 \pm 2.77$ & $6.49 \pm 2.64$ & 0.0000 & $6.16 \pm 2.78$ & $6.29 \pm 2.56$ & 0.588 \\
\hline ADL dependent, $\mathrm{n}(\%)$ & $203(39.0 \%)$ & $263(59.5 \%)$ & 0.0000 & $126(53.4 \%)$ & $124(52.5 \%)$ & 0.9195 \\
\hline IADL dependent, $n(\%)$ & $318(61.2 \%)$ & $321(72.6 \%)$ & 0.0002 & $160(67.8 \%)$ & $164(69.5 \%)$ & 0.1023 \\
\hline Cognitive impairment, $\mathrm{n}(\%)$ & $222(42.7 \%)$ & $256(57.9 \%)$ & 0.0000 & $128(54.2 \%)$ & $125(53.0 \%)$ & 0.8481 \\
\hline Number of drugs, mean \pm standard deviation & $8.22 \pm 2.75$ & $8.13 \pm 2.87$ & 0.6062 & $8.08 \pm 2.70$ & $8.08 \pm 2.99$ & 1.000 \\
\hline Overall mortality, n (\%) & $155(29.8 \%)$ & $229(51.8 \%)$ & 0.00000 & $77(33.0 \%)$ & $118(50.0 \%)$ & 0.0004 \\
\hline Total ischemic stroke, n (\%) & $26(5.0 \%)$ & $40(9.0 \%)$ & 0.01327 & $16(6.8 \%)$ & $23(9.7 \%)$ & 0.3239 \\
\hline Fatal ischemic stroke, $\mathrm{n}(\%)$ & $10(1.9 \%)$ & $18(4.1 \%)$ & 0.0425 & $6(2.5 \%)$ & $13(5.5 \%)$ & 0.1687 \\
\hline Ischemic events, other sites, n (\%) & $25(4.8 \%)$ & $25(5.7 \%)$ & 0.55467 & $12(5.1 \%)$ & $13(5.5 \%)$ & 1.0000 \\
\hline Hemorrhagic stroke/intracranial bleeding, n (\%) & $6(1.15 \%)$ & $3(0.68 \%)$ & 0.44556 & $5(2.1 \%)$ & $1(0.4 \%)$ & 0.2188 \\
\hline Major extra-cranial bleeding, n (\%) & $25(4.8 \%)$ & $15(3.4 \%)$ & 0.27357 & $13(5.5 \%)$ & $9(3.8 \%)$ & 0.4807 \\
\hline Major bleeding, total, n (\%) & $31(6.0 \%)$ & $18(4.1 \%)$ & 0.18414 & $18(7.6 \%)$ & $10(4.2 \%)$ & 0.1338 \\
\hline Fatal bleeding, n (\%) & $6(1.1 \%)$ & $5(1.1 \%)$ & 0.9738 & $4(1.7 \%)$ & $3(1.3 \%)$ & 1.0000 \\
\hline Minor bleeding, n (\%) & $37(7.1 \%)$ & $19(4.3 \%)$ & 0.06296 & $15(6.4 \%)$ & $12(5.1 \%)$ & 0.7003 \\
\hline
\end{tabular}


through the comprehensive geriatric assessment (including evaluation of comorbidity, functional and cognitive impairment, which are strong predictors of poor outcomes in elderly patients) reinforces the reliability of our findings and attenuates potential selection bias due the higher use of OAT among healthier patients.

\section{Conclusions}

Within the intrinsic limitations of an observational study, we observed an overall survival benefit of OAT over APT among older AF medical patients discharged from hospital, without increased risk of fatal and major bleedings.

\section{References}

1. Chugh SS, Havmoeller R, Narayanan K, et al. Worldwide epidemiology of atrial fibrillation: a global burden of disease 2010 Study. Circulation 2014;129:837-47.

2. Mant J, Hobbs FD, Fletcher K, et al. Warfarin versus aspirin for stroke prevention in an elderly community population with atrial fibrillation (the Birmingham Atrial Fibrillation Treatment of the Aged Study, BAFTA): a randomised controlled trial. Lancet 2007;370:493-503.

3. Olesen JB, Lip GY, Lindhardsen J, et al. Risks of thromboembolism and bleeding with thromboprophylaxis in patients with atrial fibrillation: a net clinical benefit analysis using a 'real world' nationwide cohort study. Thromb Haemost 2011;106: 739-49.

4. Friberg L, Rosenqvist M, Lip GY. Net clinical benefit of warfarin in patients with atrial fibrillation: a report from the Swedish atrial fibrillation cohort study. Circulation 2012;125:2298-307.

5. Hylek EM, D’Antonio J, Evans-Molina C, et al. Translating the results of randomized trials into clinical practice: the challenge of warfarin candidacy among hospitalized elderly patients with atrial fibrillation. Stroke 2006;37:1075-80.

6. Pugh D, Pugh J, Mead GE. Attitudes of physicians regarding anticoagulation for atrial fibrillation: a systematic review. Age Ageing 2011;40:675-83.

7. Di Pasquale G, Mathieu G, Maggioni AP, et al. Current presentation and management of 7148 patients with atrial fibrillation in cardiology and internal medicine hospital centers: the ATA AF study. Int J Cardiol 2013;167:2895-903.
8. Abdul-Rahim AH, Wong J, McAlpine C, et al. Associations with anticoagulation: a cross-sectional registry-based analysis of stroke survivors with atrial fibrillation. Heart 2014;100:557-62.

9. Bo M, Li Puma F, Badinella Martini M, et al. Health status, geriatric syndromes and prescription of oral anticoagulant therapy in elderly medical in-patients with atrial fibrillation: a prospective observational study. Int J Cardiol. 2015;187:123-5.

10. Sánchez-Barba B, Navarrete-Reyes AP, Avila-Funes JA. Are geriatric syndromes associated with reluctance to initiate oral anticoagulation therapy in elderly adults with nonvalvular atrial fibrillation? J Am Geriatr Soc 2013;61:2236-7.

11. Scowcroft AC, Lee S, Mant J. Thromboprophylaxis of elderly patients with AF in the UK: an analysis using the General Practice Research Database (GPRD) 2000-2009. Heart 2013;99:127-32.

12. van Walraven C, Hart RG, Connolly $\mathrm{S}$, et al. Effect of age on stroke prevention therapy in patients with atrial fibrillation: the atrial fibrillation investigators. Stroke 2009;40:1410-6.

13. Sardar P, Chatterjee S, Chaudhari S, Lip GY. New oral anticoagulants in elderly adults: evidence from a meta-analysis of randomized trials. J Am Geriatr Soc 2014; 62:857-64.

14. Kirchhof P, Ammentorp B, Darius H, et al. Management of atrial fibrillation in seven European countries after the publication of the 2010 ESC Guidelines on atrial fibrillation: primary results of the PREvention oF thromboembolic events-European Registry in Atrial Fibrillation (PREFER in AF). Europace 2014;16:6-14.

15. Lip GY, Laroche C, Dan GA, et al. A prospective survey in European Society of Cardiology member countries of atrial fibrillation management: baseline results of EURObservational Research Programme Atrial Fibrillation (EORP-AF) Pilot General Registry. Europace 2014;16:308-19.

16. Wolff A, Shantsila E, Lip GY, Lane DA. Impact of advanced age on management and prognosis in atrial fibrillation: insights from a population-based study in general practice. Age Ageing 2015;44:874-8.

17. Bo M, Sciarrillo I, Li Puma F, et al. Effects of oral anticoagulant therapy in medical inpatients $\geq 65$ years with atrial fibrillation. Am J Cardiol 2016;117:590-5.

18. Pamukcu B, Lip GY, Lane DA. Simplifying stroke risk stratification in atrial fibrillation patients: implications of the CHA2DS2-VASc risk stratification scores. Age Ageing 2010;39:533-5.

19. Lip GY, Frison L, Halperin JL, Lane DA.
Comparative validation of a novel risk score for predicting bleeding risk in anticoagulated patients with atrial fibrillation: the HAS-BLED (Hypertension, Abnormal Renal/Liver Function, Stroke, Bleeding History or Predisposition, Labile INR, Elderly, Drugs/Alcohol Concomitantly) score. J Am Coll Cardiol 2011;57:173-80.

20. Charlson ME, Pompei P, Ales KL, MacKenzie CR. A new method of classifying prognostic comorbidity in longitudinal studies: development and validation. J Chronic Dis 1987;40:373-83.

21. Pfeiffer E. A short portable mental status questionnaire for the assessment of organic brain deficit in elderly patients. $\mathrm{J}$ Am Geriatr Soc 1975;23:433-41.

22. Katz S, Downs TD, Cash HR, Grotz RC. Progress in development of the index of ADL. Gerontologist 1970;10:20-30.

23. Lawton MP, Brody EM. Assessment of older people: self-maintaining and instrumental activities of daily living. Gerontologist 1969;9:179-86.

24. Levey AS, Stevens LA, Schmid CH, et al. A new equation to estimate glomerular filtration rate. Ann Intern Med 2009;150:604-12.

25. Sacco RL, Kasner SE, Broderick JP, et al. An updated definition of stroke for the 21st century: a statement for healthcare professionals from the American Heart Association/American Stroke Association. Stroke 2013;44:2064-89.

26. Schulman S, Kearon C; Subcommittee on Control of Anticoagulation of the Scientific and Standardization Committee of the International Society on Thrombosis and Haemostasis. Definition of major bleeding in clinical investigations of antihemostatic medicinal products in non-surgical patients. J Thromb Haemost 2005;3:692-4.

27. Hernandez I, Baik SH, Piñera A, Zhang Y. Risk of bleeding with dabigatran in atrial fibrillation. JAMA Intern Med 2015;175:18-24.

28. Ng KH, Shestakovska O, Connolly SJ, et al. Efficacy and safety of apixaban compared with aspirin in the elderly: a subgroup analysis from the AVERROES trial. Age Ageing 2016;45:77-83.

29. Poli D, Antonucci E, Testa S, et al. Bleeding risk in very old patients on vitamin $\mathrm{K}$ antagonist treatment: results of a prospective collaborative study on elderly patients followed by Italian Centres for Anticoagulation. Circulation 2011;124:824-9.

30. Perysinaki GS, Theodorakopoulou V, Bertsias A, et al. Almost half of octogenarians and nonagenarians admitted acutely to internal medicine ward die during admission or within 6 months after discharge: time to redefine treatment goals? J Am Geriatr Soc 2015;63:380-3. 